\title{
A NOTE ON LANGUAGE AND TRANSLATION
}

\begin{abstract}
All translations from Swahili unless otherwise noted are mine. All interviews referred to in the text were conducted in Swahili between January 2014 and July 2015. To protect my interlocutors' anonymity, I use pseudonyms. Swahili, including words derived from Arabic, is given in Standard Swahili Spelling (Kiswahili Sanifu).
\end{abstract}



Disputing Discipline 
